\title{
Effect of pulse wave shape on Taylor cone jetting behavior in electrohydrodynamics micro jet process
}

\author{
Bo Xing ${ }^{1, a}$, Chuncheng Zuo ${ }^{1,2, b}$, Yebo Lu ${ }^{1, c}$, Fengli Huang ${ }^{1, d}$, \\ Libing Zhang ${ }^{1, e}$
}

${ }^{1}$ School of Mechanical and Electrical Engineer, Jiaxing university, Jia xing, 314001, China ${ }^{2}$ Key Laboratory of special purpose Equipment and Advanced Manufacturing Technology, Ministry of Education \& Zhejiang Province, Zhejiang University of Technology, Hang zhou, 310000, China axblut@163.com, ${ }^{b} z u o c c @ m a i l . z j x u . e d u . c n,{ }^{c} l u y e b o @ m a i l . z j x u . e d u . c n, ~{ }^{d}$ windon416@163.com, ${ }^{\mathrm{e}}$ libi nzhan@126.com

Keywords: Pulse wave shape; Taylor cone jetting; jetting behavior; Meniscus

\begin{abstract}
The effect of pulse wave shape on Taylor cone jetting behavior in electrohydrodynamics micro jet process has been investigated by using the ethyl alcohol solution. The square wave, trapezoid wave and triangular wave with different amplitude have been used in the experiment. The sequential images gathered by the charge-coupled camera combined with a strobe LED have been used to present cone jetting behavior with respect to the time. The shapes of meniscus under different voltage condition have been examined during the jetting process. Results indicated that when the square wave and the trapezoid wave were used the activation of cone jetting and its duration time were closely relied on the amplitude voltage and its duration time, thus this is beneficial for the accurate control of droplet frequency and volume in the drop on demand printing process. However, the introduction of positive amplitude resulted in the delay of the followed cone jetting thus creates error for the jetting uniformity control. The results also indicated that triangular wave was failed to induce the jetting thus this kind of wave shape is not suitable for the jetting control in electrohydrodynamics printing process.
\end{abstract}

\section{Introduction}

Nowadays, with the increasing requirement of flexible and wearable electronics, the printed electronics technology has been attracted extensive attention in the production of sensors, display device, photovoltaic cells and other electronics. Among the current printed electronics technologies, ink jet printing technology is one of the effective and flexible methods due to its advantages such as low cost, contactless with substrate, easy realization of digitization and the ability for complicated pattern preparation $^{[1]}$. Electrohydrodynamics micro printing is an alternative inkjet process which employs electrical force induced by the electrical field between nozzle and substrate to drag ink droplet from nozzle onto substrate to deposit a desired pattern. This electrical force induced inkjet process offers many advantages such as high resolution printing, better compatibility of ink, low cost and good maneuverability ${ }^{[2]}$.

To control the formation and ejection of droplet in a "drop on demand" manner is the key to improve the process controllability and the printing quality. Pulse voltage is usually applied to realize the droplet on demand control in the EHD printing process ${ }^{[3]}$. However, some parameters such as amplitude and duration time of the pulse always result in varied electrical behavior between ink and substrate thus result in different ejection behavior ${ }^{[4]}$. Therefore, it is possible to clarify the effect of pulse voltage parameters on the jetting feature in the printing process. In this paper, different pulse voltage shape with varied amplitude, duration time and rising time were designed to investigate the effect of pulse voltage on the cone jetting behavior in the EHD process, this is of significant importance for the realization of EHD printing in the production of micro electronics. 


\section{Experiment}

Figure1shows the experiment set up used in this research. The DC power supply was connected to nozzle and the maximum output voltage is $10 \mathrm{KV}$. The power amplifier and the arbitrary function generator were connected to substrate and the negative voltage was applied. The soft tube was used to connect air pump, vial and nozzle connected. The vial height was controlled to adjust the ink supply pressure and the height was set as $1 \mathrm{~mm}$ (distance between the vial bottom and substrate surface). The nozzle was a 34G stainless fluid dispensing head and its inner diameter is 60um. The substrate is a glass slip with the size of $50 \mathrm{~mm} \times 25 \mathrm{~mm}$ and was located on the substrate table. The distance between the nozzle and substrate was adjusted to 150um. The moving speed of the substrate was set as $1 \mathrm{~mm} / \mathrm{s}$. The micro in situ observation system was composed of lens, ccd and led. The CCD camera with strobe LEDs was used to observe the meniscus in inkjet heads. Self-developed software was used to extract and measure the profile of meniscus under different jetting conditions. The ink used in this experiment was ethyl alcohol solution.

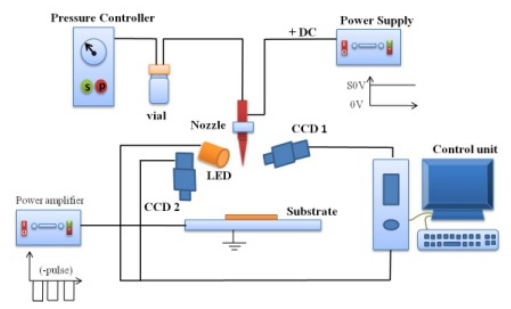

Figure 1. EHD printing setup in the experiment

\section{Results and discussion}

In this work, the dc voltage applied on nozzle was kept as the constant value thus the meniscus formed on nozzle tip but there is no jetting. And then the pulse voltage of $750 \mathrm{~V}$ was applied on the substrate and this amplitude is sufficient to induce the cone jetting when the pulse shape was suitably designed. Figure 2 shows the meniscus feature when the square wave was used. The first wave was a negative pulse square type and the second is the square wave with periodic negative and positive amplitude. Figure 2a shows that the meniscus deformed at the amplitude voltage and the cone jet appeared after 50us with the duration of the pulse. The cone jetting disappeared with the ending of pulse and the followed jetting was occurred at the next pulse and delay time is also 50us. Figure $2 \mathrm{~b}$ shows the evolution of meniscus height based on the pulse time. It can be found that the height of meniscus increased to the maximum 30us later when the amplitude voltage was applied and the height has no obvious change till the end of the followed pulse. Figure 2c shows that the cone jetting feature in the first negative pulse is similar to figure $2 \mathrm{a}$ and there is no jetting occurred at the followed positive pulse. However, at the subsequent negative pulse the cone jetting appeared 150us later when the amplitude voltage was applied. This result indicated that the added positive pulse voltage delayed the deformation behavior of meniscus and the jetting was suppressed. From figure $2 \mathrm{~d}$, it can be found that the height of meniscus increased to its maximum with the first negative pulse then retune to its original and was stretched again with the followed negative pulse. 


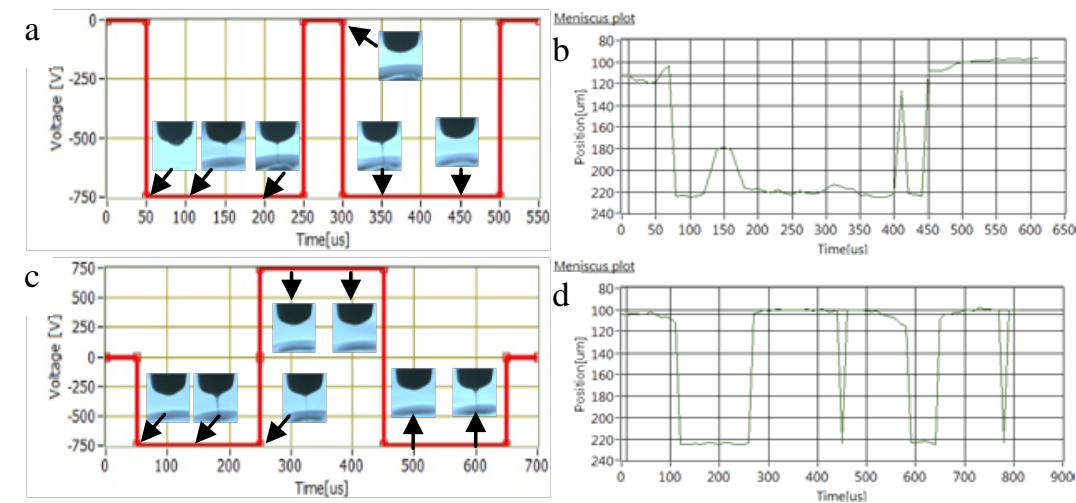

Figure 2. The meniscus feature on different time when the square wave was applied $(\mathrm{a}, \mathrm{c})$ and the evolution of meniscus height (b, d).

Figure 3 show the jetting behavior and the meniscus height evolution during the pulse period of the trapezoid wave. From figure 3a it can be found there is no jetting in the rising stage of the amplitude voltage and the jetting occurred 100us later when the amplitude voltage was arrived. There is also no jetting in the falling stage of the trapezoid wave. In the subsequent pulse, the jet appeared 100us later when the amplitude voltage was appeared and the jetting behavior is similar to the first pulse. From figure 3b, it can be found that the meniscus deformed to 135um in the first pulse and then further deformed to 150um in the followed pulse. Figure 3c shows that when the trapezoid wave with periodic positive and negative wave was applied, the jetting in the first negative was occurred at 250us and ended at 300us. In the positive pulse stage there is also no jetting occurred. However, it also can be found that there is a 150us delay for the next jetting in the followed negative pulse. Figure $3 \mathrm{~d}$ shows that the deformation of meniscus is closely depend on the amplitude voltage but compared with figure $3 \mathrm{~b}$ it can be found that the maximum height of the meniscus with the adding of positive pulse is less than the condition that without positive pulse.
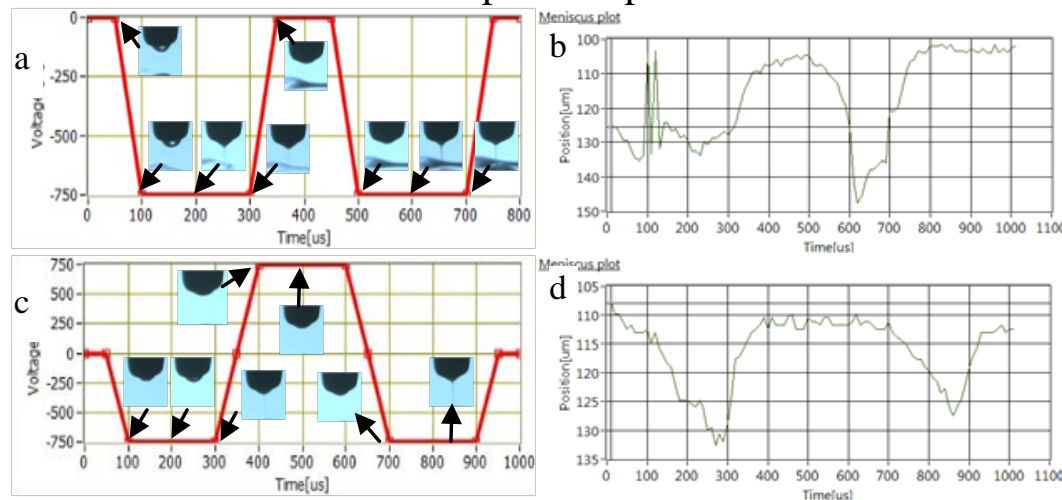

Figure 3. The meniscus feature on different time when the trapezoid wave was used (a, c) and the evolution of meniscus height (b, d).

Figure 4 shows the jetting behavior and the meniscus height evolution during the pulse period of the triangular wave. An interesting phenomenon is that from figure 4 a and c we can found that although there is meniscus deformation occurred during the negative pulse of the triangular wave, there was no jetting was observed in the whole pulse period. From figure $4 \mathrm{~b}$ and $\mathrm{d}$ it can be found that there was obvious deformation of meniscus with respect to time, this implies the amplitude voltage is a main factor for the meniscus deformation but the duration of pulse is essential for the jetting trigger as the charge in the meniscus need time to accumulate till the electrical force applied on tip of the meniscus is large enough to conquer the surface tension of the meniscus. Therefore, when considering on the controllability and the frequency of the jetting during printing process the square without adverse pulse is recommend to apply in the droplet on demand control in the EHD printing process. 


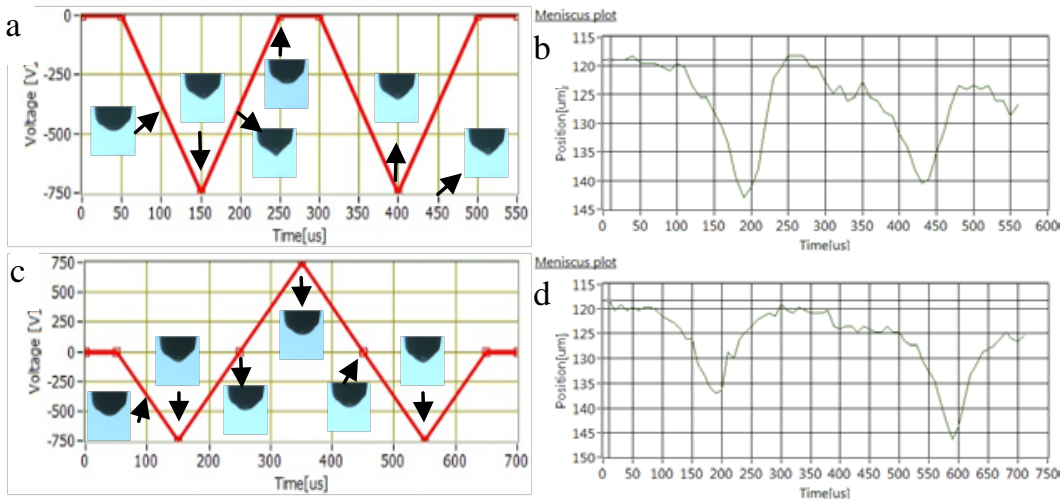

Figure 4. The meniscus feature on different time when the triangular wave was used (a, c) and the evolution of meniscus height (b, d).

\section{Summary}

(1) The amplitude voltage of the pulse voltage is important because the deformation of meniscus is closely depend on this voltage but the introduction of adverse pulse resulted in the delay of meniscus deformation thus the jetting was also retarded;

(2) The trapezoid wave caused rising and falling time of the amplitude voltage and this is an alternative factor for the control of jetting amount, but the square wave is beneficial to produce a consistent pulsating for the pulse voltage singal;

(3) The application of triangular wave resulted in the deformation of meniscus but the jetting was not induced in the whole period due to the inefficient duration time for charge accumulation.

\section{Acknowledgement}

This work was financial supported by the zhejiang provincial natural science foundation (NO. LZ14E050002) and Zhejiang province public welfare technology application research project (NO.2015C31069) and scientific research project of Zhejiang Provincial Department of Education (NO.Y201534088) and zhejiang provincial natural science foundation (NO. EM2015042001).

\section{References}

[1] X. Lei, S. Daoheng, Electrohydrodynamic printing under applied pole-type nozzle configuration, Appl. Phys. Lett, 102(2013)024101.

[2]T. Leo,B. Kira, Airflow assisted printhead for high-resolution electrohydrodynamic jet printing onto non-conductive and tilted surfaces, Appl. Phys. Lett, 107(2015) 054103

[3] K. Kye-Si, L. Dae-Yong, Investigation of pulse voltage shape effectson electrohydrodynamic jets using a vision measurement technique, J Micromech Microeng, 23(2013)1-12.

[4] T. Leo, B. Kira, Airflow assisted printhead for high-resolution electrohydrodynamic jet printing onto non-conductive and tilted surfaces, Appl. Phys. Lett, 107(2015)054103. 